\title{
A new constraint for chondrule formation: condition for the rim formation of barred-olivine textures
}

\author{
Hitoshi Miura ${ }^{1}$, Etsuro Yokoyama ${ }^{2}$, Ken Nagashima $^{3}$, Katsuo Tsukamoto ${ }^{1}$, and Atul Srivastava ${ }^{1}$ \\ ${ }^{1}$ Department of Earth and Planetary Materials Science, Graduate School of Science, Tohoku University, \\ Aoba 6-3, Aramaki, Aoba-ku, Sendai 980-8578, Japan \\ ${ }^{2}$ Computer Centre, Gakushuin University, Mejiro 1-5-1, Toshima-ku, Tokyo 171-8588, Japan \\ ${ }^{3}$ Division of Electrical, Electronic and Information Engineering, Graduate School of Enginnering, \\ Osaka Univerisity, Suita 565-0871, Japan
}

(Received October 18, 2010; Revised June 3, 2011; Accepted June 3, 2011; Online published February 2, 2012)

\begin{abstract}
A barred-olivine (BO) chondrule usually has an olivine rim that covers the chondrule surface. Numerous experiments have been carried out to reproduce the $\mathrm{BO}$ texture. However, the rim structure could be reproduced only in a few studies reported in the literature. The difficulty in reproducing the rim structure lies in the fact that its formation condition has not been constrained experimentally or theoretically. In the present paper, we have carried out numerical simulations of crystal growth of a highly-supercooled melt droplet of pure forsteritic composition $\left(\mathrm{Mg}_{2} \mathrm{SiO}_{4}\right)$, and succeeded in reproducing the double structure, i.e. the rim and the dendrite. The droplet cools from the surface, the temperature of which should be cooler than the center of the droplet. Since a crystal grows faster along the cooler surface than across the hotter center, the rim was found to be formed when the temperature difference between the center of the droplet and its surface is large enough. From our results, both from numerical simulations and analytical consideration, we found that the double structure of rim and the dendrite could be formed only when the cooling rate is within a narrow range, which depends upon the degree of supercooling. Our results, for the first time, could explain why the formation of rim of BO texture was hardly reproduced in the previous experiments reported in the literature to date.
\end{abstract}

Key words: Chondrule solidification texture, melt growth, supercooling, hypercooling, phase-field simulation.

\section{Introduction}

Chondrules are millimeter-sized, spherical-shaped grains containing olivine, pyroxene, metal, sulfide, and glass with igneous textures. They are considered to have been formed from molten droplets about 4.6 billion years ago in the solar nebula (Amelin et al., 2002); it is believed that they melted and cooled again to solidify in a short period of time (Sorby, 1877; Nelson et al., 1972; Tsuchiyama et al., 1980; Lofgren and Russell, 1986; Jones and Lofgren, 1993; Osada and Tsuchiyama, 2001; Tsuchiyama et al., 2004). Since chondrules possess about 80 vol.\% of chondritic meteorites in the most abundant cases (Jones et al., 2000), they must carry information about the early history of our solar system. Chondrules have various textures commonly described as porphyritic, barred, and radial textures; all of these textures appear for the same chondrule bulk composition (Lux et al., 1981; Lofgren and Russell, 1986). Many authors have carried out dynamic crystallization experiments to constrain the formation condition of each texture (Hewins et al., 2005 and references therein), however, the formation mechanism is not yet fully understood.

Barred-olivine (BO) chondrules are characterized by parallel set(s) of olivine bars in a thin section (Weisberg, 1987).

Copyright (C) The Society of Geomagnetism and Earth, Planetary and Space Sciences (SGEPSS); The Seismological Society of Japan; The Volcanological Society of Japan; The Geodetic Society of Japan; The Japanese Society for Planetary Sciences; TERRAPUB.

doi:10.5047/eps.2011.06.004
It has been considered that olivine bar crystals are actually platy in three-dimensions (Tsuchiyama et al., 2000; Noguchi, 2002). A BO chondrule usually has an olivine rim that covers the chondrule surface. This olivine rim has the same crystallographic orientation as inner olivine platelets. The olivine rim has not been reproduced in early dynamic crystallization experiments except in a limited number of runs (Lofgren and Lanier, 1990; Radomsky and Hewins, 1990). Tsukamoto et al. $(1999,2001)$ succeeded in reproducing the rim structure from a forsterite melt droplet in a container-less crystallization experiment using an aeroacoustic levitation technique. They found from their in-situ observation that the droplet cooled very rapidly at a rate of $R_{\text {cool }} \approx 100-1000 \mathrm{~K} \mathrm{~s}^{-1}$, and then crystallized at a large supercooling of $\Delta T \approx 600 \mathrm{~K}$ within a very short period of time (less than $1 \mathrm{~s}$ ). However, they reproduced the rim structure only in a few cases, so the formation condition was not constrained from their experiments. On the other hand, Tsuchiyama et al. (2004) also succeeded in reproducing the rim structure by evaporation in vacuum. The cooling rate was $R_{\text {cool }}=1000 \mathrm{~K} \mathrm{hr}^{-1}$, which is much slower than that of the container-less experiments by about three orders of magnitude. They did not observe the solidification process of the sample in-situ, so detailed information such as the timing of nucleation, the solidification timescale, and crystal growth pattern were not available. They considered that the rim was formed by the rapid crystal growth along the droplet surface, which should become cooler than the in- 
terior because of the latent heat of evaporation. However, their hypothesis has not been verified yet.

To clarify the formation condition of the rim, the crystallization process inside the chondrule melt droplet should be investigated. We carried out numerical simulations of crystallization of a highly-supercooled melt droplet with a pure forsteritic $\left(\mathrm{Mg}_{2} \mathrm{SiO}_{4}\right)$ composition (Miura et al., 2010). We considered the situation that a tiny crystal seeded at the droplet surface triggers crystallization of the droplet. We found that the rapid crystal growth along the droplet surface occurs when the cooling rate is very large. However, we did not investigate over a wide range of supercooling of the droplet in the previous paper.

The aim of this paper is to clarify the formation condition of the rim structure. We carry out numerical simulations for a wide range of supercooling and cooling rate by using the phase-field method, which is one of the most effective numerical methods to simulate crystal growth in a supercooled liquid. As a first step, we consider the situation of container-less crystallization experiments using levitation methods (Tsukamoto et al., 1999, 2001; Nagashima et al., 2006, 2008; Srivastava et al., 2010) because in these experiments the thermal profile of the droplet and the crystal growth pattern were observed in-situ, so we can verify the results of our numerical simulations. For comparison, we consider a droplet of pure forsteritic $\left(\mathrm{Mg}_{2} \mathrm{SiO}_{4}\right)$ composition, which was adopted in these experiments.

Glass formation, which is expected to occur when a melt droplet cools very rapidly, is not taken into consideration in this paper. Tangeman et al. (2001) reported the first synthesis of a forsterite $\left(\mathrm{Mg}_{2} \mathrm{SiO}_{4}\right)$ composition glass using containerless conditions. Nagashima et al. (2008) summarized the condition for glass formation in a $\mathrm{Mg}_{2} \mathrm{SiO}_{4}-\mathrm{MgSiO}_{3}$ system for various cooling rates (see their figure 4). Under levitated conditions, a pure forsterite melt becomes a glass when it cools at $R_{\text {cool }} \approx 300 \mathrm{~K} \mathrm{~s}^{-1}$ or faster. In contrast, under non-levitated conditions, an extremely large cooling rate of $R_{\text {cool }} \approx 10^{6}-10^{7} \mathrm{~K} \mathrm{~s}^{-1}$ is required for glass formation because of the heterogeneous nucleation on the contact area (e.g., sample holder). This suggests that glass formation from a pure forsteritic melt is very difficult once nucleation occurs.

We describe the basic equation of the phase-field method in Section 2, where the basic concept of the phase-field method is summarized in Appendix A. In Section 3, we show the results of the calculation, in which various crystal growth patterns appear inside the droplet. In Section 4, we discuss the conditions for rapid crystal growth along the droplet surface and propose a new constraint for chondrule formation, especially, for barred-olivine chondrules. We present some conclusions in Section 5.

\section{Method}

\subsection{Basic equations}

We employ the phase-field method for the crystallization of a pure material from its melt (model I in Wang et al., 1993). In their model, the solid and liquid phases were distinguished by $\phi=0$ and $\phi=1$, respectively. We adopt the same basic equations as those used in our previous paper, in which the cooling term at the droplet surface was taken into consideration (Miura et al., 2010). The time evolutions of phase $\phi$ and temperature $T$ are given by

$$
\frac{W}{\mu} \frac{\partial \phi}{\partial t}=\frac{\sqrt{2}}{12} \frac{T_{\mathrm{M}}}{T} p^{\prime}(\phi)\left(T-T_{\mathrm{M}}\right)-\frac{T_{\mathrm{M}} \Gamma}{4 W} g^{\prime}(\phi)+T_{\mathrm{M}} \Gamma W \nabla^{2} \phi
$$

and

$$
\frac{\partial}{\partial t} \int_{\mathrm{V}}[c T+L p(\phi)] d \mathrm{v}=\oint_{\mathrm{A}-\mathrm{A}_{\mathrm{s}}} \kappa \boldsymbol{\nabla} T \cdot \boldsymbol{n} d \mathrm{a}-\oint_{\mathrm{A}_{\mathrm{s}}} q_{\mathrm{s}} d \mathrm{a}
$$

respectively, where $\mu$ is the kinetic coefficient (see Eq. (A.5)), $T_{\mathrm{M}}$ is the equilibrium melting point, $\Gamma \equiv \sigma / L$ is the capillary length, $\sigma$ is the interfacial free energy between crystal and liquid, $L$ is the latent heat of crystallization per unit volume, $W$ is the thickness of the solidliquid interface, $c$ is the volumetric heat capacity per unit volume, and $\kappa$ is the thermal conductivity. Functions $p(\phi)$ and $g(\phi)$ are given by $p(\phi)=\phi^{3}\left(10-15 \phi+6 \phi^{2}\right)$ and $g(\phi)=\phi^{2}(1-\phi)^{2}$, respectively, and the prime indicates the derivative by $\phi$. Equation (2) can be obtained by integrating a time-dependent thermal diffusion equation over a small volume element $\mathrm{V}$ having a boundary $\mathrm{A}$ and then applying Gauss's theorem (Miura et al., 2010). $A_{\mathrm{s}}$ is an intersection of the boundary A and the droplet surface if such a surface exists. $\boldsymbol{n}$ is the outward pointing unit normal of the boundary $d$ a. The second term of Eq. (2) represents the energy loss at the droplet surface caused by thermal radiation, thermal conduction with ambient nebula gas, and latent heat of evaporation, where $q_{\mathrm{s}}$ is the net heat flux.

Although one could introduce anisotropies in $\mu$ and $\sigma$ to express an anisotropic interface and to simulate facet formation (McFadden et al., 1993; Uehara and Sekerka, 2003), for simplicity we do not take anisotropy into account.

The governing equation of phase $\phi$, Eq. (1), was derived from an entropy function to satisfy the entropy production being positive in any small volume element (Wang et al., 1993). The details of the derivation will be found in the reference. We briefly describe the physical meaning of Eq. (1) in Appendix A.

\subsection{Numerical scheme}

Chondrules are three-dimensional spherules, so a threedimensional calculation is desirable. However, this requires an unrealistically large number of computational nodes for a chondrule-sized object $\left(\sim 10^{9} \mu \mathrm{m}^{3}\right)$. To reduce the computational cost, we consider a two-dimensional disk in the $x y$-plane (circle but not a sphere), assume uniformity in the $z$-direction and drop the derivative by $z$. Figure 1 shows a schematic picture of the square mesh used for computation. We adopt a computational domain of $-250 \leq x \leq 250 \mu \mathrm{m}$ and $-250 \leq y \leq 250 \mu \mathrm{m}$ to consider a droplet of $250 \mu \mathrm{m}$ in radius, and discretized by a $1000 \times 1000$ square mesh (mesh sizes for $x$ - and $y$-directions are $\Delta_{x}=\Delta_{y}=0.5$ $\mu \mathrm{m})$. Other numerical procedures are the same as Miura $e t$ al. (2010).

We carried out a convergence test with smaller mesh sizes of $\Delta_{x}=\Delta_{y}=0.25 \mu \mathrm{m}$, and found that the calculation result was not modified except for minor changes in the shapes of dendrite tips.

We put a seed crystal at the droplet surface to trigger the crystal growth of the supercooled droplet. At the beginning 


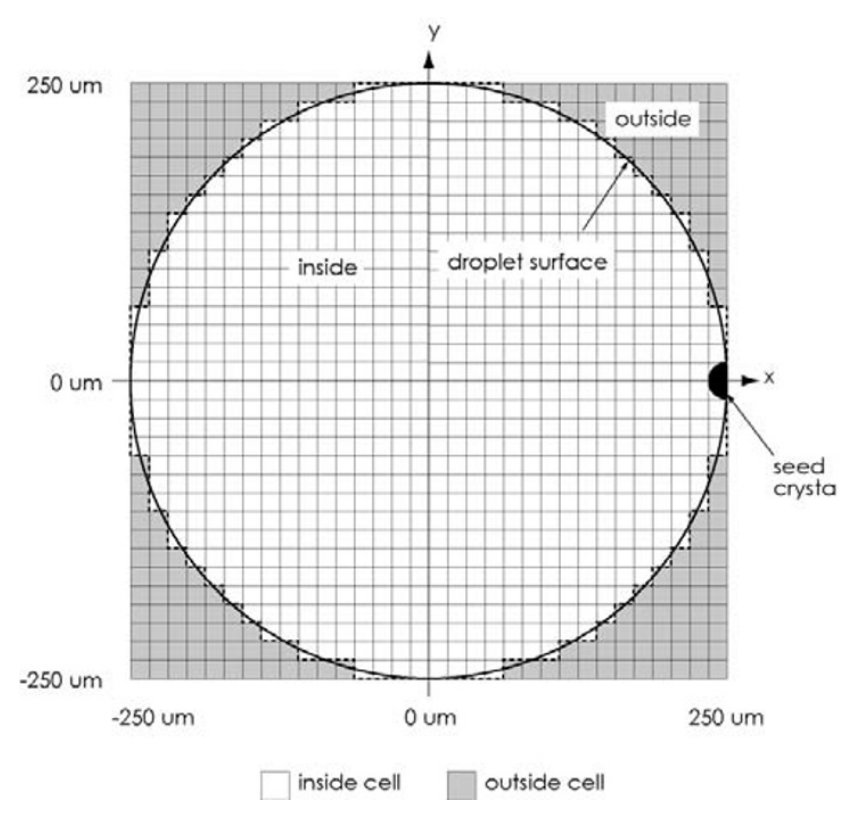

Fig. 1. Two-dimensional square mesh for computation. The thick solid curve is the actual surface of droplet. The computational cells inside (white) and outside (gray) the actual surface are defined by whether positions of their centers locate the inside or outside, respectively. The computational surface (thick dashed lines) is the mesh boundaries between inside cells and outside cells. The crystal growth is triggered by a seed crystal at the droplet surface.

of the calculation, we substitute $\phi=0$ only at the seeding point without any change in temperature (see Fig. 2(a), for example). The radius of the seed crystal is assumed to be $10 \mu \mathrm{m}$.

\subsection{Initial temperature profile}

In this paper, we suppose that a completely-molten melt droplet cools rapidly from the surface. Due to the surface cooling, the droplet surface is cooler than the center. The temperature profile inside the droplet is given by an analytic solution of the thermal diffusion equation assuming a uniform cooling ( $\partial T / \partial t$ does not depend on $r$ ) and a constant value of $q_{\mathrm{s}}$ (Miura et al., 2010). The analytic solution is given by

$$
T_{0}(r)=T_{\mathrm{s}, 0}+\delta T_{\mathrm{c}-\mathrm{s}, 0}\left[1-\left(\frac{r}{r_{\mathrm{d}}}\right)^{2}\right],
$$

where $T_{\mathrm{s}, 0}$ is the temperature at the droplet surface, $r_{\mathrm{d}}$ is the droplet radius, and

$$
\delta T_{\mathrm{c}-\mathrm{s}, 0}=\frac{q_{\mathrm{s}} r_{\mathrm{d}}}{2 \kappa_{\mathrm{L}}}
$$

is the temperature difference between the center and surface of the droplet with the thermal conductivity of the liquid phase equal to $\kappa_{\mathrm{L}}$. The subscript " 0 " means $t=0$, namely, at the time when the seeding occurs.

\subsection{Physical properties of forsterite sample}

In this study, we consider the sample of pure forsteritic composition. The physical properties we adopted are as follows; $c=5.7 \times 10^{7} \mathrm{erg} \mathrm{cm}^{-3} \mathrm{~K}^{-1}, L=2.4 \times 10^{10} \mathrm{erg}$ $\mathrm{cm}^{-3}, T_{\mathrm{M}}=2163 \mathrm{~K}$ (Nagashima et al., 2006, 2008), $\kappa_{\mathrm{C}}=$ $5.0 \times 10^{5} \mathrm{erg} \mathrm{cm}^{-1} \mathrm{~K}^{-1} \mathrm{~s}^{-1}$ (Pertermann and Hofmeister,
2006), $\kappa_{\mathrm{L}}=2.0 \times 10^{5} \mathrm{erg} \mathrm{cm}^{-1} \mathrm{~K}^{-1} \mathrm{~s}^{-1}$ (Moriya, 1963), $\sigma=620 \mathrm{erg} \mathrm{cm}^{-2}$ (Tanaka et al., 2008), $\mu=0.4 \mathrm{~cm}$ $\mathrm{s}^{-1} \mathrm{~K}^{-1}$ (Tsukamoto et al., 1999, 2001; Nagashima et al., 2006), and $W=0.25 \mu \mathrm{m}$ (Murray et al., 1995), where $\kappa_{\mathrm{C}}$ is the thermal conductivity of the crystal phase. These parameters are the same as those adopted in Miura et al. (2010).

\subsection{Input parameters}

2.5.1 Heat flux at droplet surface $q_{\mathrm{s}}$ Let us estimate the heat flux $q_{\mathrm{s}}$ at the droplet surface from containerless crystallization experiments (Tsukamoto et al., 1999; Nagashima et al., 2006, 2008). Samples were melted during levitation using a $\mathrm{CO}_{2}$ laser and then quenched by turning off the laser (Tsukamoto et al., 1999). In this case, the droplet cools at a maximum rate due to the thermal radiation and the heat conduction by the gas-jet (Nagashima et al., 2008). The cooling rate can be controlled to be slower by adjusting the output power of the laser (Nagashima $e t$ al., 2006).

The net heat flux at the droplet surface determines the cooling rate of the droplet $R_{\text {cool }}$. The heat flux $q_{\mathrm{s}}$ is calculated by (Miura et al., 2010)

$$
q_{\mathrm{s}}=c r_{\mathrm{d}} R_{\mathrm{cool}} / 3 \text {. }
$$

In Table 1, we summarize the cooling rate $R_{\text {cool }}$ and the heat flux $q_{\mathrm{s}}$ expected in container-less experiments. In this table, we use $c$ of a pure forsteritic melt (see Section 2.4). The typical heat flux is found to be $q_{\mathrm{s}} \approx 10^{8}-10^{9} \mathrm{erg} \mathrm{cm}^{-2}$ $\mathrm{s}^{-1}$. Based on Table 1, we adopt the following values; $q_{\mathrm{s}}=5 \times 10^{8}, 1 \times 10^{9}, 2 \times 10^{9}, 5 \times 10^{9}, 1 \times 10^{10} \mathrm{erg} \mathrm{cm}^{-2} \mathrm{~s}^{-1}$. The smallest value is the same order of magnitude to that estimated from the container-less experiments. The reason why we consider larger $q_{\mathrm{s}}$ values is to see how the growth pattern changes at such an extremely large cooling rate.

2.5.2 Supercooling at droplet surface $\Delta \boldsymbol{T}_{\mathrm{s}, 0}$ The molten droplet becomes largely supercooled by a few hundred $\mathrm{K}$ or more in the container-less environment (Nelson et al., 1972; Tsukamoto et al., 1999; Nagashima et al., 2006, 2008; Tanaka et al., 2008). Tsukamoto et al. (1999) carried out container-less crystallization experiments by using an aero-acoustic levitator and observed a forsterite melt droplet being supercooled by $\Delta T \approx 600 \mathrm{~K}$ at a cooling rate of $R_{\text {cool }} \approx 400 \mathrm{~K} \mathrm{~s}^{-1}$. Nagashima et al. (2006) adopted a gasjet levitator and observed a forsterite melt droplet being supercooled by $\Delta T \approx 1000 \mathrm{~K}$ at a cooling rate of $R_{\text {cool }} \approx 100$ $\mathrm{K} \mathrm{s}^{-1}$. Tanaka et al. (2008) modeled the homogeneous nucleation and sequential crystal growth inside a supercooled melt droplet based on classical nucleation theory and found that a forsterite melt droplet should be supercooled by about $1000 \mathrm{~K}$ even if it cools at a much smaller cooling rate, e.g., $R_{\text {cool }}=10^{-2} \mathrm{~K} \mathrm{~s}^{-1}$.

In this paper, we suppose that a forsterite melt droplet never nucleates homogeneously at a relatively low supercooling $(\Delta T<600 \mathrm{~K})$, so the crystallization is triggered by a collision with a micron-sized crystal (seeding). Depending on the timing of the seeding, crystallization occurs at various values of supercooling. In this paper, we adopt values of the supercooling at the droplet surface when the seeding occurs of $\Delta T_{\mathrm{s}, 0}=200 \mathrm{~K}, 300 \mathrm{~K}, 400 \mathrm{~K}, 500 \mathrm{~K}$, and $600 \mathrm{~K}$. 
Table 1. Cooling rate $R_{\text {cool }}$ of molten droplet in container-less crystallization experiments using levitation methods. We read $R_{\text {cool }}$ from each cooling curve obtained at each experiment just before the droplet crystallized. We calculate the heat flux $q_{\mathrm{s}}$ and the initial temperature difference between the center and surface of the droplet $\delta T_{\mathrm{c}-\mathrm{s}, 0}$ by using Eqs. (5) and (4), respectively. Refs: [1] Tsukamoto et al. (2001), [2] Nagashima et al. (2006), [3] Nagashima et al. (2008).

\begin{tabular}{|c|c|c|c|c|c|c|}
\hline $\begin{array}{c}\text { Sample } \\
\text { composition }\end{array}$ & $\begin{array}{c}\text { Radius } \\
r_{\mathrm{d}}[\mu \mathrm{m}]\end{array}$ & $\begin{array}{l}\text { Cooling rate } \\
R_{\text {cool }}\left[\mathrm{K} \mathrm{s}^{-1}\right]\end{array}$ & $\begin{array}{c}\text { Heat flux } \\
q_{\mathrm{s}}\left[\mathrm{erg} \mathrm{cm}^{-2} \mathrm{~s}^{-1}\right]\end{array}$ & $\begin{array}{l}\text { Temp. diff. } \\
\delta T_{\mathrm{c}-\mathrm{s}, 0}[\mathrm{~K}]\end{array}$ & $\begin{array}{c}\text { Levitation } \\
\text { method }\end{array}$ & Ref \\
\hline forsterite & $\approx 1000$ & $\approx 350$ & $\approx 6.7 \times 10^{8}$ & $\approx 170$ & aero-acoustic & [1] \\
\hline forsterite & $\approx 1000$ & $\approx 50$ & $\approx 1.0 \times 10^{8}$ & $\approx 25$ & gas-jet & [2] \\
\hline forsterite & $\approx 1000$ & $\approx 70$ & $\approx 1.3 \times 10^{8}$ & $\approx 10$ & gas-jet & [3] \\
\hline $\mathrm{Mg}_{1.8} \mathrm{SiO}_{3.8}$ & $\approx 1000$ & $\approx 30-40$ & $\approx(0.6-0.8) \times 10^{8}$ & $\approx 5$ & gas-jet & [3] \\
\hline
\end{tabular}

Here, we introduce a hypercooling limit $\Delta T_{\text {hyp }}$ defined by (Glicksman and Schaefer, 1967; Herlach, 1994);

$$
\Delta T_{\text {hyp }}=L / c .
$$

The crystallization process from a highly supercooled melt changes drastically when the supercooling exceeds $\Delta T_{\text {hyp }}$. When $\Delta T<\Delta T_{\text {hyp }}$, crystallization takes place by a twostep process. Once nucleation has initiated solidification, the subsequent growth of the solid phase leads to the release of the latent heat of crystallization. In the case of rapid crystal growth, a steep rise in temperature will occur, termed recalescence, which takes place under nearadiabatic conditions. After recalescence, some fraction of the liquid phase still remains. The remaining liquid will solidify under near-equilibrium conditions as the latent heat is removed. The latter step was termed a "filling-in" process (Chalmers, 1964). On the other hand, when $\Delta T>\Delta T_{\text {hyp }}$, the supercooled melt solidifies exclusively during recalescence, so there is no remaining liquid because the droplet temperature during recalescence does not exceed the melting point. The hypercooling limit of a pure forsterite melt is $\Delta T_{\text {hyp }}=425 \mathrm{~K}$ (Nagashima et al., 2006).

\section{Result}

\subsection{Dendritic growth}

Figure 2 shows a result of phase-field simulation for $\Delta T_{\mathrm{s}, 0}=200 \mathrm{~K}\left(T_{\mathrm{s}, 0}=1963 \mathrm{~K}\right)$ and $q_{\mathrm{s}}=5 \times 10^{8} \mathrm{erg} \mathrm{cm}^{-2}$ $\mathrm{s}^{-1}$. This is a gray contour map of the phase value ranging from $\phi=0$ (solid phase, black) to $\phi=1$ (liquid phase, white). The transition layer between the solid and liquid is too thin to distinguish in this map, meaning that the solidliquid interface is very sharp. At the beginning, there is a tiny seed crystal on the right hand side at the droplet surface (panel (a)). At $0.02 \mathrm{~s}$ after seeding, a dendritic crystal grows from the seed crystal (panel (b)). The temperature distribution is shown by isothermal lines in terms of $T-T_{\mathrm{M}}$. Crystal growth is accompanied by release of the latent heat of crystallization, so the temperature around the dendritic crystal increases up to almost the melting point. On the other hand, the supercooling far from the dendritic crystal is still large, resulting in a steep temperature gradient ahead of the growing dendrite tips. The dendritic crystal is spreading into the entire region inside the droplet (panel (c)). Around $0.06 \mathrm{~s}$ after seeding, the rapid dendritic crystal growth comes to a stop because the temperature inside the droplet increases to the melting point (panel (d)). It is found that a liquid phase still remains at the gap between dendrite tips. Finally, the remaining liquid solidifies completely as the latent heat of
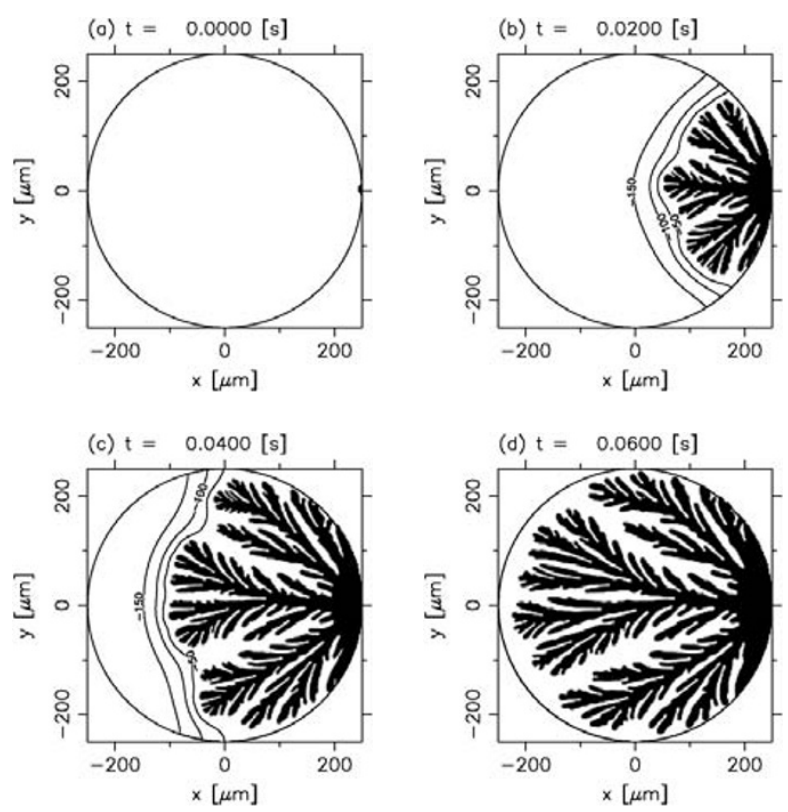

Fig. 2. Result of phase-field simulation for $\Delta T_{\mathrm{s}, 0}=200 \mathrm{~K}$ and $q_{\mathrm{s}}=5 \times 10^{8} \mathrm{erg} \mathrm{cm}^{-2} \mathrm{~s}^{-1}$. A circle of $250 \mu \mathrm{m}$ in radius is the external shape of droplet and the target of the calculation is only inside the circle. Black and white regions represent crystal and liquid phases, respectively. The temperature distribution is shown by contours with labels indicating $T-T_{\mathrm{M}}$, namely, negative and positive labels mean supercooled and above melting point, respectively. The interval of the isotherms is $50 \mathrm{~K}$. The initial condition (a), and $t=0.02 \mathrm{~s} \mathrm{(b),} 0.04 \mathrm{~s}$ (c), and $0.06 \mathrm{~s}(\mathrm{~d})$ after seeding.

crystallization is removed by the surface cooling. It took $0.42 \mathrm{~s}$ to solidify completely. The luminosity of the droplet increases rapidly from panel (a) to (d), and then decreases gradually (Miura et al., 2010).

\subsection{Dendritic growth with rim structure}

Figure 3 shows a result for $\Delta T_{\mathrm{s}, 0}=300 \mathrm{~K}\left(T_{\mathrm{s}, 0}=1863\right.$ $\mathrm{K})$ and $q_{\mathrm{s}}=2 \times 10^{9} \mathrm{erg} \mathrm{cm}^{-2} \mathrm{~s}^{-1}$. At the beginning, one can see concentric isothermal lines of $T_{0}-T_{\mathrm{M}}=-250 \mathrm{~K}$ and $-200 \mathrm{~K}$ (panel (a)), meaning that there is a large temperature difference between the center and surface of the droplet. The temperature difference calculated by Eq. (4) is $\delta T_{\mathrm{c}-\mathrm{s}, 0}=125 \mathrm{~K}$, so the temperature at the droplet center $\left(T_{0}-T_{\mathrm{M}}=-175 \mathrm{~K}\right)$ is $175 \mathrm{~K}$ below the melting point. At $0.008 \mathrm{~s}$ after seeding, a dendritic crystal grows from a seed crystal as well, as shown in Fig. 2(b) (panel (b)). At $0.016 \mathrm{~s}$ after seeding, it is found that the dendritic crystal growing across the droplet center is suppressed compared with Fig. 2(c) (panel (c)). At $0.024 \mathrm{~s}$ after seeding, the crys- 

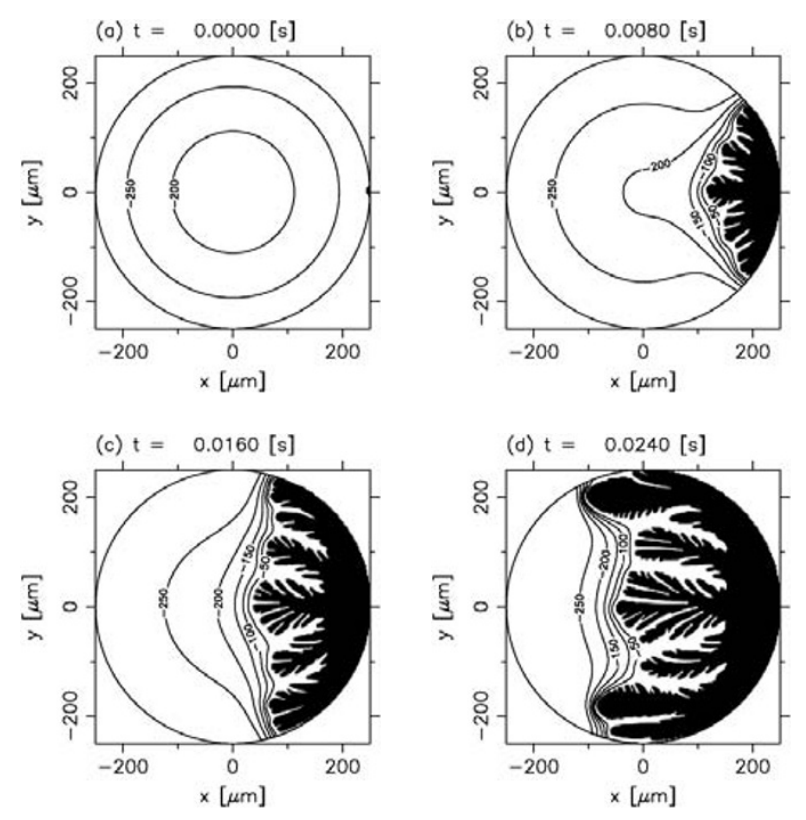

Fig. 3. Result of phase-field simulation for $\Delta T_{\mathrm{s}, 0}=300 \mathrm{~K}$ and $q_{\mathrm{s}}=2 \times 10^{9} \mathrm{erg} \mathrm{cm}^{-2} \mathrm{~s}^{-1}$. The initial condition (a), and $t=0.008$ $\mathrm{s}(\mathrm{b}), 0.016 \mathrm{~s}(\mathrm{c})$, and $0.024 \mathrm{~s}$ (d) after seeding. Others are the same as Fig. 2.

tal is growing rapidly along the droplet surface (panel (d)). The crystal along the droplet surface is one of the branches of the dendritic crystal, and it seems to be surrounding the droplet like the rim of a BO chondrule.

\subsection{Rim growth}

Figure 4 shows a result for $\Delta T_{\mathrm{s}, 0}=400 \mathrm{~K}\left(T_{\mathrm{s}, 0}=1763\right.$ $\mathrm{K})$ and $q_{\mathrm{s}}=1 \times 10^{10} \mathrm{erg} \mathrm{cm}^{-2} \mathrm{~s}^{-1}$. One can see dense concentric isotherms at the beginning (panel (a)). The temperature difference between the center and surface is calculated to be $\delta T_{\mathrm{c}-\mathrm{s}, 0}=625 \mathrm{~K}$ from Eq. (4). The inner most isotherm is $T_{0}-T_{\mathrm{M}}=200 \mathrm{~K}$, indicating a temperature above the melting point. The crystal growth pattern is completely different from Figs. 2 and 3. No dendritic crystal grows inside the droplet (panel (b)). The crystal grows only along the droplet surface where the temperature is below the melting point. At $0.016 \mathrm{~s}$ after seeding, almost of all of the droplet surface is covered with the crystal (panel (c)). There is liquid remaining inside the crystal rim (panel (d)). The remaining liquid gradually solidifies from the outside to the inside as the latent heat of crystallization is removed through the shell crystal.

\subsection{Parallel dendritic growth}

Figure 5 shows a result for $\Delta T_{\mathrm{s}, 0}=500 \mathrm{~K}\left(T_{\mathrm{s}, 0}=1663\right.$ $\mathrm{K})$ and $q_{\mathrm{s}}=2 \times 10^{9} \mathrm{erg} \mathrm{cm}^{-2} \mathrm{~s}^{-1}$. The temperature difference between the center and surface of the droplet is calculated as $\delta T_{\mathrm{c}-\mathrm{s}, 0}=125 \mathrm{~K}$, so the temperature at the center $\left(T_{0}-T_{\mathrm{M}}=-375 \mathrm{~K}\right)$ is $375 \mathrm{~K}$ below the melting point. Note that the temperature distribution can be divided into two regions; a hypercooled region along the surface, and normally supercooled at the central region, where "normally" means that the supercooling does not exceed the hypercooling limit $\Delta T_{\text {hyp }}$ (see Eq. (6)). The seed crystal grows isotropically at first (panel (b)). However, when the crystal-liquid interface
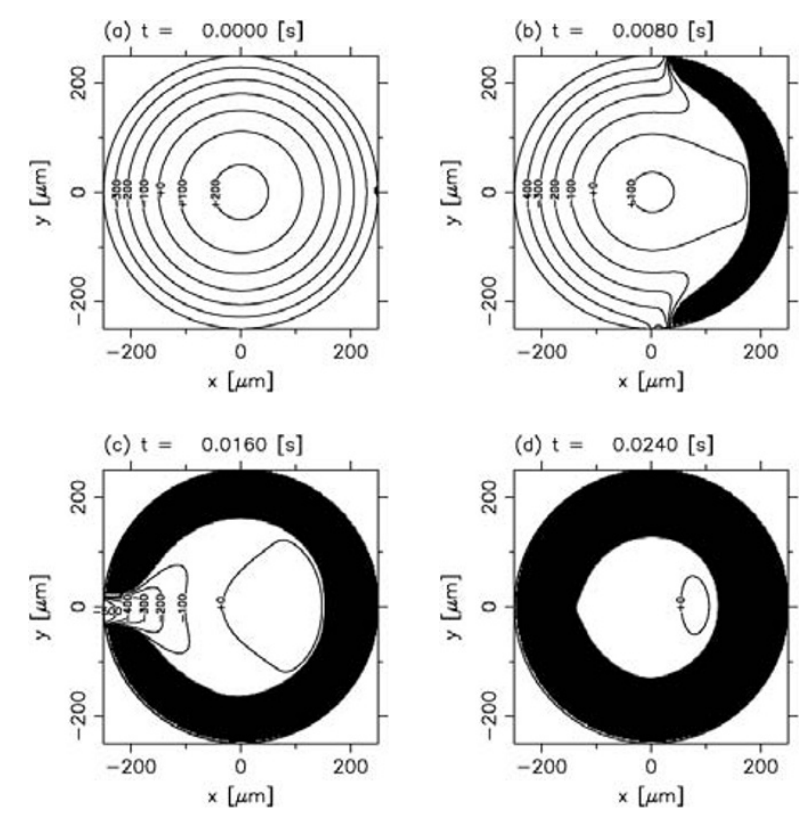

Fig. 4. Result of phase-field simulation for $\Delta T_{\mathrm{s}, 0}=400 \mathrm{~K}$ and $q_{\mathrm{s}}=1 \times 10^{10} \mathrm{erg} \mathrm{cm}^{-2} \mathrm{~s}^{-1}$. The interval of the isotherms is 100 $\mathrm{K}$. The initial condition (a), and $t=0.008 \mathrm{~s} \mathrm{(b),} 0.016 \mathrm{~s}$ (c), and 0.024 $\mathrm{s}$ (d) after seeding. Others are the same as Fig. 2.
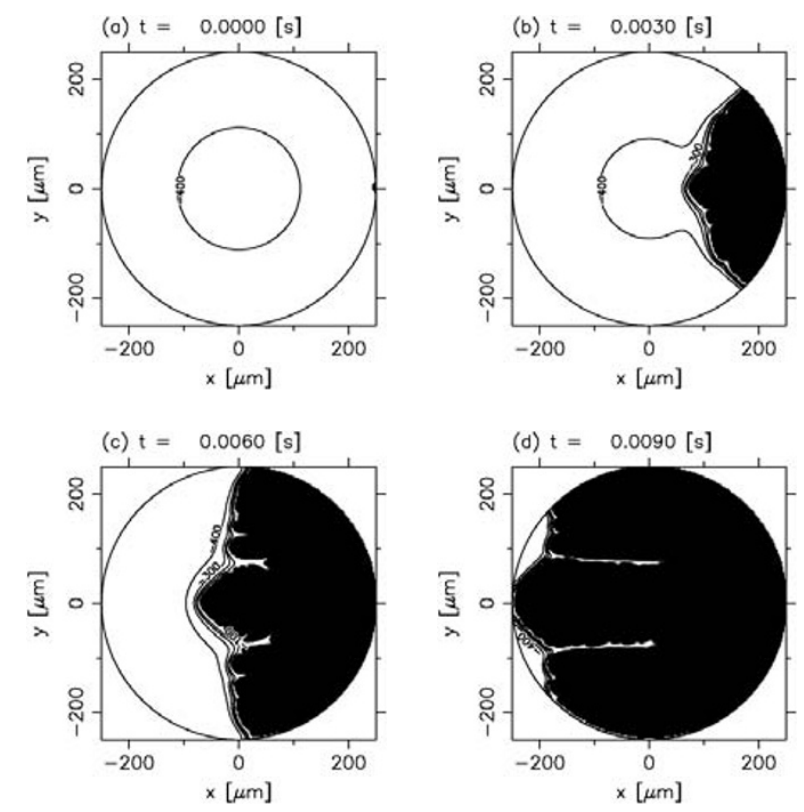

Fig. 5. Result of phase-field simulation for $\Delta T_{\mathrm{s}, 0}=500 \mathrm{~K}$ and $q_{\mathrm{s}}=2 \times 10^{9} \mathrm{erg} \mathrm{cm}^{-2} \mathrm{~s}^{-1}$. The interval of the isotherms is $100 \mathrm{~K}$. The initial condition (a), and $t=0.003 \mathrm{~s} \mathrm{(b),} 0.006 \mathrm{~s}$ (c), and $0.009 \mathrm{~s}$ (d) after seeding. Others are the same as Fig. 2.

reaches the normally supercooled region, it splits into some parallel branches (panel (c)). After the tips of the branches pass the normally supercooled region, it is found that a liquid phase remains among the branches (panel (d)). The remaining liquid solidifies as the latent heat of crystallization is removed. 

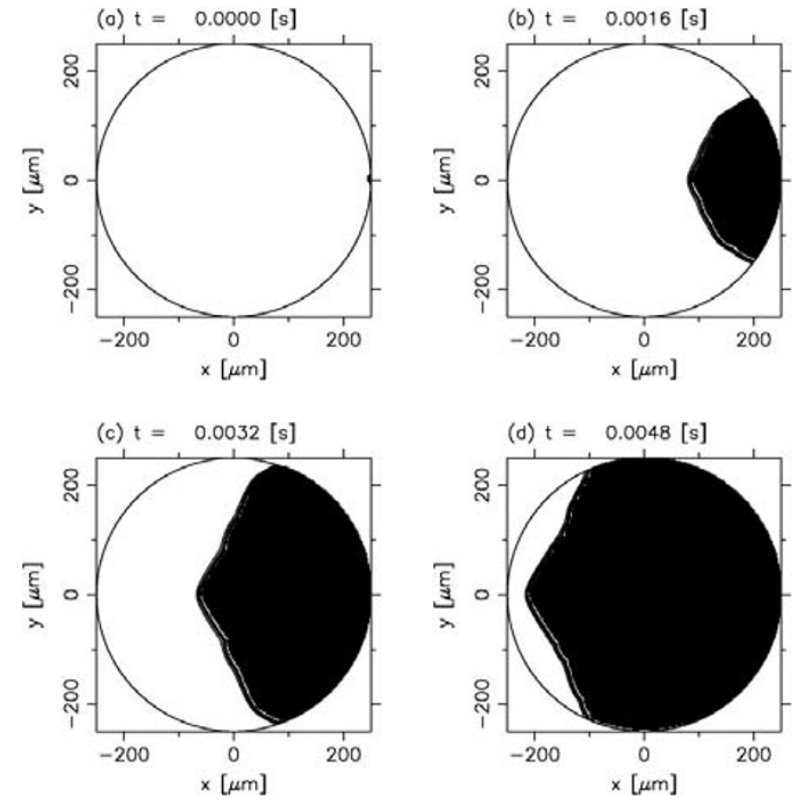

Fig. 6. Result of phase-field simulation for $\Delta T_{\mathrm{s}, 0}=600 \mathrm{~K}$ and $q_{\mathrm{s}}=5 \times 10^{8} \mathrm{erg} \mathrm{cm}^{-2} \mathrm{~s}^{-1}$. The interval of the isotherms is $100 \mathrm{~K}$. No labels indicating $T-T_{\mathrm{M}}$ is displayed for the purpose of visualization. The initial condition (a), and $t=0.0016 \mathrm{~s} \mathrm{(b),} 0.0032 \mathrm{~s} \mathrm{(c),} \mathrm{and}$ $0.0048 \mathrm{~s}(\mathrm{~d})$ after seeding. Others are the same as Fig. 2.

\subsection{Isotropic growth}

Figure 6 shows a result for $\Delta T_{\mathrm{s}, 0}=600 \mathrm{~K}\left(T_{\mathrm{s}, 0}=1563\right.$ $\mathrm{K})$ and $q_{\mathrm{s}}=5 \times 10^{8} \mathrm{erg} \mathrm{cm}^{-2} \mathrm{~s}^{-1}$. The temperature difference between the center and surface of the droplet is only $\delta T_{\mathrm{c}-\mathrm{s}, 0} \approx 30 \mathrm{~K}$. The temperature at the center $\left(T_{0}-T_{\mathrm{M}}=\right.$ $-570 \mathrm{~K})$ is $570 \mathrm{~K}$ below the melting point, so the entire droplet is hypercooled. From panels (b)-(d), one can see that the crystal grows isotropically from the seed crystal. There is no remaining liquid after the crystal growth front has passed because the liquid can solidify completely without removal of the latent heat of crystallization. The shape of the crystal-liquid interface is quasi-planar, which denotes macroscopic planarity of the interface, but on the microscopic scale of the heat-flow the interface does not necessarily behave as a plane front (Glicksman and Schaefer, 1967).

\section{Discussion}

\subsection{Constraint on cooling rate for rim formation}

Here, we derive the constraint on the cooling rate for rim formation based on the discussion of Miura et al. (2010).

Let us consider crystal growth from a seed crystal at one side of the droplet surface to the opposite side. There are two possible routes for crystal growth; along the droplet surface and across the center. For the former case, the distance of the growth route is the longest, however, the growth velocity is the fastest because the surface should be cooler than the center due to surface cooling. Here, we assume that the growth velocity of the crystal is $V\left(\Delta T_{0}\right) \propto \Delta T_{0}^{\beta}$, where $\Delta T_{0}$ is the supercooling inside the droplet when the seeding occurs. The index is $\beta \approx 2.5-3.5$ according to the dendrite growth theory (Langer and Müller-Krumbhaar, 1978; Xu, 1998). Hereinafter, we adopt $\beta=3$ as a typical value. The growth timescale along the droplet surface $\tau_{\mathrm{s}}$ is given by di- viding the arc distance $\pi r_{\mathrm{d}}$ by the growth velocity $V\left(\Delta T_{\mathrm{s}, 0}\right)$ as

$$
\tau_{\mathrm{s}}=\frac{\pi r_{\mathrm{d}}}{V\left(\Delta T_{\mathrm{s}, 0}\right)}
$$

where we consider a constant supercooling of $\Delta T_{\mathrm{s}, 0}$, namely, a constant growth velocity along the droplet surface. On the other hand, the growth velocity across the droplet center is not constant because of the temperature gradient inside the droplet (see Eq. (3)). The growth timescale across the center $\tau_{\mathrm{c}}$ is given by the following integral form;

$$
\tau_{\mathrm{c}}=2 \int_{0}^{r_{\mathrm{d}}} \frac{d r}{V\left(\Delta T_{0}(r)\right)}=\frac{2 r_{\mathrm{d}}}{V\left(\Delta T_{\mathrm{s}, 0}\right)} I(\beta, \alpha),
$$

where

$$
\alpha \equiv \frac{\delta T_{\mathrm{c}-\mathrm{s}, 0}}{\Delta T_{\mathrm{s}, 0}}, \quad I(\beta, \alpha) \equiv \int_{0}^{1} \frac{d \tilde{r}}{\left[\alpha\left(\tilde{r}^{2}-1\right)+1\right]^{\beta}},
$$

$r$ is the distance from the droplet center, and $\tilde{r}=r / r_{\mathrm{d}}$. The condition for rapid crystal growth along the droplet surface is given by $\tau_{\mathrm{s}}<\tau_{\mathrm{c}}$, which is rewritten as

$$
I(\beta, \alpha)>\frac{\pi}{2} .
$$

By integrating $I(\beta, \alpha)$ numerically, we obtain (Miura et al., 2010)

$$
\alpha>\sim 0.2, \quad \text { for } \beta=3 .
$$

The cooling rate is rewritten as $R_{\text {cool }}=6 \alpha \kappa_{\mathrm{L}} \Delta T_{\mathrm{s}, 0} / c r_{\mathrm{d}}^{2}$ by using Eqs. (4), (5), and (9). Substituting Eq. (11) into the expression for the cooling rate, we obtain the formation condition of the rim as

$$
R_{\text {cool }}>\sim 2000\left(\frac{\Delta T_{\mathrm{s}, 0}}{300 \mathrm{~K}}\right)\left(\frac{r_{\mathrm{d}}}{250 \mu \mathrm{m}}\right)^{-2} \mathrm{~K} \mathrm{~s}^{-1} \text {. }
$$

It should be noted that the condition given by Eq. (11) or (12) is applicable only when $\tau_{\mathrm{s}}<\tau_{\text {cool }}$, where $\tau_{\text {cool }}$ is the cooling timescale of the droplet (Miura et al., 2010). If not, the droplet temperature drops considerably during solidification, so Eq. (3) cannot be used in this analysis. We can apply the condition given by Eq. (11) or (12) to our results because the typical growth timescale of $\tau_{\mathrm{s}}<0.1 \mathrm{~s}$ is shorter than the cooling timescale of $\tau_{\text {cool }}>0.1 \mathrm{~s}$.

\subsection{Condition to produce each growth pattern}

Figure 7 shows the calculation conditions and the resultant crystal growth patterns on a $q_{\mathrm{s}}-T_{\mathrm{s}, 0}$ diagram. The top border is $\delta T_{\mathrm{c}-\mathrm{s}, 0}$ calculated by Eq. (4). The right border is the normalized supercooling at the droplet surface defined by (Glicksman and Schaefer, 1967)

$$
\Delta \theta_{\mathrm{s}, 0}=\frac{T_{\mathrm{M}}-T_{\mathrm{s}, 0}}{\Delta T_{\mathrm{hyp}}} .
$$

Note that the droplet surface is hypercooled when $\Delta \theta_{\mathrm{s}, 0}>$ 1 (see Eq. (6)). Filled symbols indicate the calculation conditions in which the rim was formed (Section 3.1, 3.2, and 3.3). Two solid curves in Fig. 7 show the conditions of $\alpha=0.2$ and $\alpha=1$, respectively; the former is the criterion for rim formation, and the latter corresponds to the condition above which the temperature at the droplet 


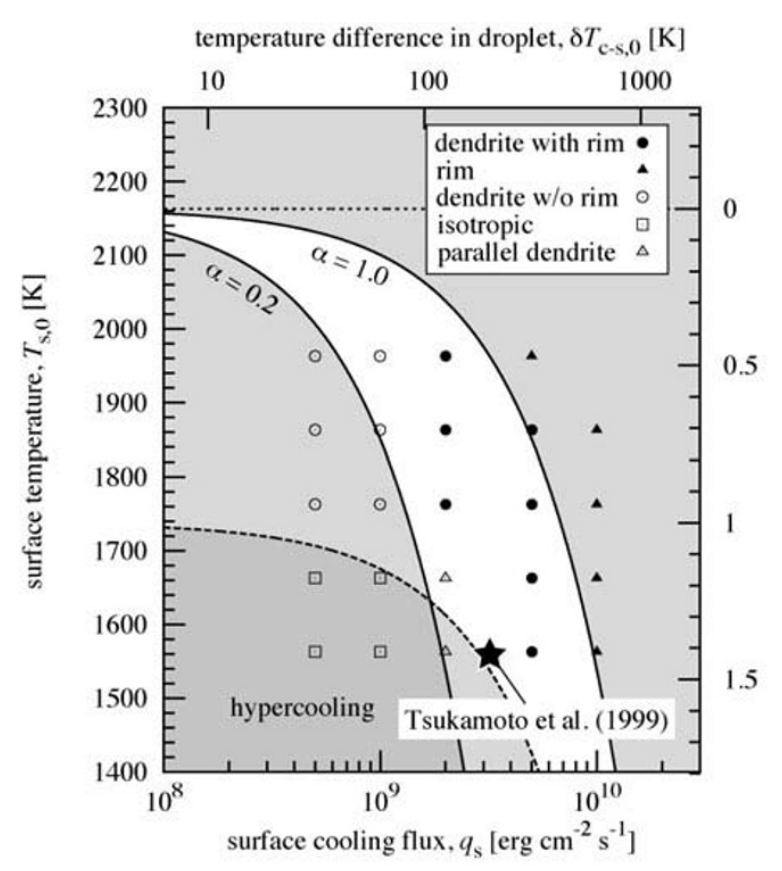

Fig. 7. Calculation conditions and the resultant crystal growth patterns on $q_{\mathrm{s}}-T_{\mathrm{s}, 0}$ diagram. $\delta T_{\mathrm{c}-\mathrm{s}, 0}$ is the temperature difference between the surface and center of the droplet. $\theta_{\mathrm{s}}=\left(T_{\mathrm{M}}-T_{\mathrm{s}, 0}\right) / \Delta T_{\mathrm{hyp}}$ is the normalized supercooling at the droplet surface, where $\Delta T_{\text {hyp }}$ is the hypercooling limit. The solid curves correspond to the conditions of $\alpha=0.2$ and $\alpha=1$, respectively, where $\alpha=\delta T_{\mathrm{c}-\mathrm{s}, 0} / \Delta T_{\mathrm{s}, 0}$ is the normalized temperature difference. The dashed curve is the criterion below which the entire droplet is hypercooled.

center exceeds the melting point (see Eq. (11)). The rim was found to be formed at conditions to the upper right of the $\alpha=0.2$ curve. From comparison with the results of phase-field simulations, we conclude that the condition given by Eq. (11) or (12) are plausible as the criterion for rim formation.

The dashed curve is the parameter space below which the entire droplet is hypercooled. It is found that isotropic growth (see Section 3.5) occurred at conditions below the dashed curve.

A large star symbol indicates the condition of the container-less crystallization experiment by Tsukamoto et al. (1999), in which the rim surrounding parallel sets of olivine bars was successfully reproduced from a pure forsteritic composition melt droplet. In their experiment, the droplet crystallized at a supercooling of $\Delta T_{\mathrm{s}, 0} \approx 600$ $\mathrm{K}$, which gives the normalized surface undercooling of $\Delta \theta_{\mathrm{s}, 0} \approx 1.4$. The temperature difference inside the droplet was estimated as $\delta T_{\mathrm{c}-\mathrm{s}, 0} \approx 170 \mathrm{~K}$ (see Table 1 ), which gives $\alpha \approx 0.4$. Since this experimental condition satisfied the condition for rim formation, their sample having the rim structure is a reasonable result.

We consider the experimental condition of Tsuchiyama et al. (2004). In their experiment, the cooling rate of the droplet was $R_{\text {cool }}=1000 \mathrm{~K} \mathrm{hr}^{-1}$ if we assume that it is the same as the cooling rate of the furnace. The temperature difference between the center and surface of the droplet can be estimated as $\delta T_{\mathrm{c}-\mathrm{s}, 0} \approx 0.02 \mathrm{~K}$ for a sample radius of $r_{\mathrm{d}}=500 \mu \mathrm{m}$ and with typical thermodynamic properties of silicates $\left(c=3 \times 10^{7} \mathrm{erg} \mathrm{K}^{-1} \mathrm{~cm}^{-3}\right.$ and $\kappa_{\mathrm{L}}=2 \times 10^{5}$ erg $\mathrm{cm}^{-1} \mathrm{~s}^{-1} \mathrm{~K}^{-1}$, Murase and McBirney, 1973). From the condition of Eq. (11), the molten sample in their experiment must have crystallized at very low supercooling as $\Delta T_{\mathrm{s}, 0}<0.1 \mathrm{~K}$ to form the rim. The nucleation at such very small supercooling might be due to the contact of the droplet to the carbon capsule. However, the exact value of the nucleation supercooling was not measured, so we cannot test our new constraint with their experiment. Insitu temperature measurement during droplet solidification is required to clarify the condition of rim formation.

\subsection{Implication for chondrule solidification textures}

The numerical model that we adopted in this study was used to simulate the crystal growth process in a supercooled melt droplet of a single composition, namely, the composition of the crystal phase is the same as that of the parent liquid phase. However, real chondrules are multi-component, namely, the composition of the crystal phase is not necessarily the same as the bulk composition of the parent liquid (e.g., Jones, 1990). Therefore, not only the temperature distribution inside the droplet, but also the partitioning and diffusion of elements near the crystal-liquid interface (Watson, 1996, 2004) should be taken into consideration. To elucidate the formation mechanism of chondrule solidification textures, it is required to model the crystallization process of a multi-component melt droplet at a largely undercooled state (e.g., Bi and Sekerka, 1998, 2002).

Before the multi-component modeling begins, we make some predictions for chondrule solidification textures based on our single-component phase-field calculations. In our model, the droplet after complete solidification is just a single crystal spherule, probably with no solidification texture remaining inside. However, if the droplet contains small amounts of incompatible elements in olivine, such as $\mathrm{Ca}$ and $\mathrm{Al}$, these elements tend to partition into the remaining liquid phase during crystal growth (Libourel, 1999; Pack and Palme, 2003). The inhomogeneity in elemental composition should relate to the solidification texture. Therefore, the distribution of the remaining liquid phase inside the droplet has some implications for the formation mechanism of chondrule solidification textures.

To visualize the distribution of the remaining liquid phase, we introduce a crystallization timescale $\tau_{\text {cry }}$ into our phase-field calculation. The timescale $\tau_{\text {cry }}$ shows how long the liquid takes to solidify at a certain position, and it is calculated by

$$
\tau_{\text {cry }}^{(i, j)}=t_{0.1}^{(i, j)}-t_{0.9}^{(i, j)},
$$

where $t_{0.1}^{(i, j)}$ and $t_{0.9}^{(i, j)}$ are the times when $\phi_{i, j}=0.1$ and 0.9 , respectively, at the grid point $(i, j) . t_{0.9}^{(i, j)}$ means the time when the liquid at the grid point $(i, j)$ begins to crystallize, and $t_{0.1}^{(i, j)}$ means the time when the grid point is almost completely solidified. The remaining liquid phase should have large $\tau_{\text {cry }}$ because it solidified very slowly.

Figure 8 is the gray-scale map of $\tau_{\text {cry }}$ for each result of the phase-field simulation shown in Figs. 2-6. The gray map shows the value of $\tau_{\text {cry }}$ on a logarithmic scale; darker gray for rapidly crystallized regions and lighter gray for more slowly crystallized ones. It is found that the shape of the darker gray region corresponds to the crystal growth pattern for each phase-field simulation. In panels (d) and (e), there 

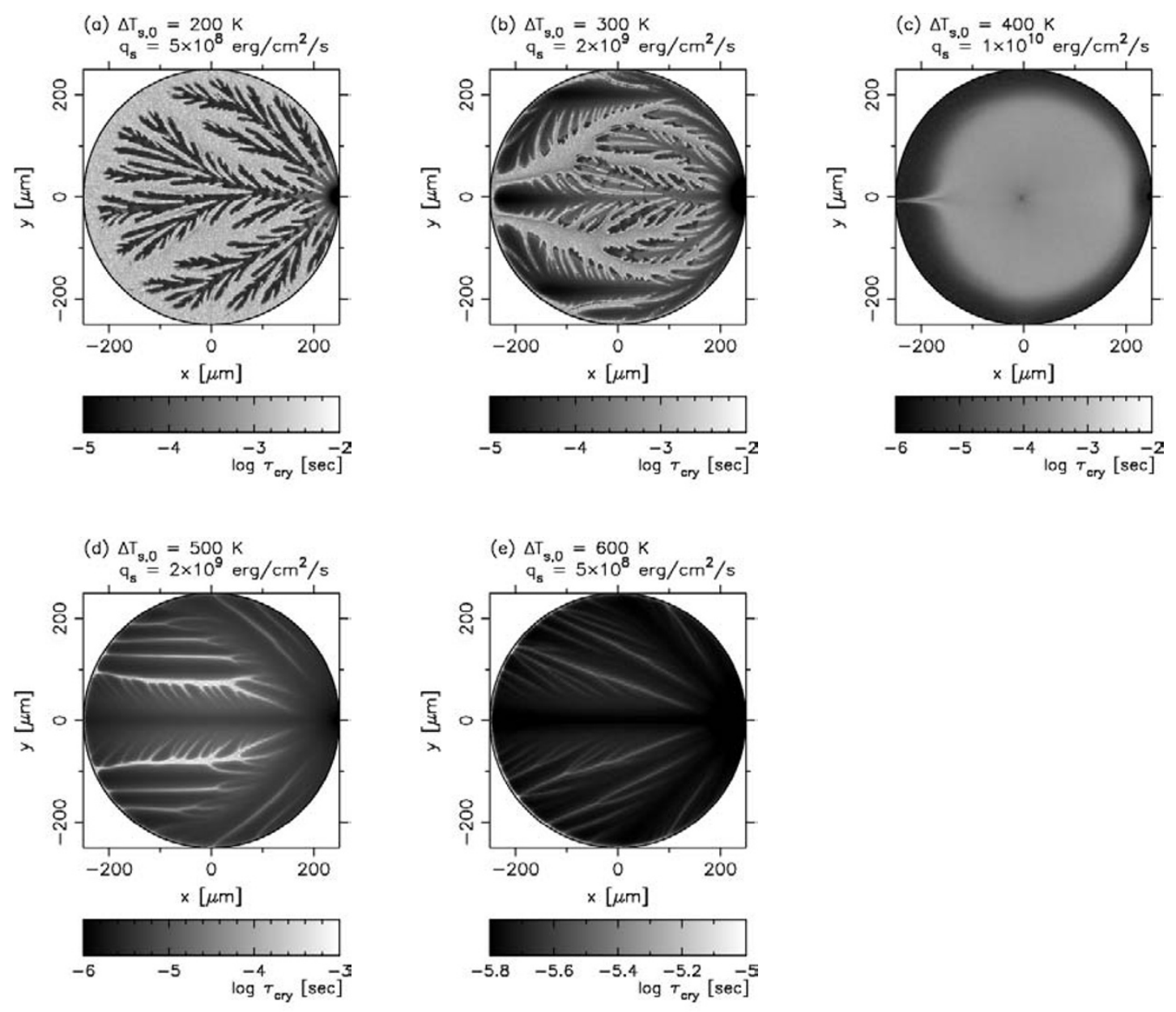

Fig. 8. Distribution of crystallization timescale $\tau_{\text {cry }}$ in a completely solidified droplet. Panels (a)-(e) correspond to Figs. 2-6, respectively. Note that the range of gray scale are different in each panel.

are some light gray lines that were not clearly identified in Figs. 5 and 6, respectively. These lines correspond to positions of some dents at the quasi-planar crystal-liquid interface. As shown in Figs. 5 and 6, the interface is not a complete plane but has some small undulations. During the advance of the interface, the released latent heat of crystallization poured into the dents by thermal diffusion even at the hypercooled state. The temperature at the dents becomes slightly higher than the ambient region, making the growth speed slower. Therefore, the track of the dent position remains on the map as the region where the liquid solidified more slowly.

The gray-scale map of $\tau_{\text {cry }}$ just visualized the difference of crystal growth timescale during solidification. The relationship to the chondrule solidification textures was not clear. However, we consider that the chondrule solidification textures reflect the crystal growth pattern inside the droplet. Our phase-field simulations are the first step to understand the formation mechanism of chondrule solidification textures.

\section{Conclusion}

The chondrule solidification texture reflects the crystal growth pattern inside the melt droplet at the time of forma- tion in the early solar nebula. We numerically investigated the following two thermal effects on the crystal growth pattern; (i) the cooling at the droplet surface and (ii) the release of latent heat of crystallization. Surface cooling makes the droplet surface cooler than the center. Since the crystal growth rate is faster along the cooler surface than across the hotter center, the rim was formed when the cooling rate is large enough. The release of the latent heat of crystallization caused a rapid temperature increase near the growing rim, resulting in a very steep temperature gradient at the interface between the rim and the remaining liquid (melt) phase. This "reversed" temperature gradient led to dendrite formation due to the morphological instability. We found that the double structure of rim and dendrite could be formed only when the cooling rate is within a narrow range. An understanding of these thermal effects on chondrule melt solidification is the first step to elucidate the formation mechanism of chondrule solidification textures.

Acknowledgments. We thank Dr. H. Kimura for his kind encouragement to submit this paper. We are grateful to Prof. Y. Inatomi for useful discussion on the results of our phase-field simulations. We acknowledge helpful comments from Prof. Joseph A. Nuth and an anonymous referee. H. M. was supported by Tohoku University Global COE Program "Global Education and Research Center for 
Earth and Planetary Dynamics". This research was partially supported by the Ministry of Education, Science, Sports and Culture, Grant-in-Aid for Scientific Research (A), 22244066, 2010-2013.

\section{Appendix A. Phase-Field Method (PFM)}

The classical approach to the modeling of first order phase transformations involves tracking the free boundary that separates the growing phase (e.g., crystal) from the parent phase (e.g., supercooled liquid). PFM provides an alternative approach, according to which a new variable, the phase field $\phi$, is introduced to keep track of the phase, taking on constant values indicative of each of the bulk phases and making a transition between these values over a thin transition layer that plays the role of the classically sharp interface. One of the advantages of PFM is not to require explicit tracking of the free boundary. This makes the numerical model simpler than the classical approach.

In the PFM developed by Wang et al. (1993), the crystallization process can be simulated by solving the coupled equations for the phase field and temperature field given by Eqs. (1) and (2), respectively. The latter is easily recognized as the time-dependent thermal diffusion equation taking into account the release of latent heat of crystallization, as explained in the text.

The physical meaning of Eq. (1) except for the diffusion term is that the phase value $\phi(x, t)$ changes to minimize the Helmholtz free energy density $f(T(\boldsymbol{x}, t), \phi(\boldsymbol{x}, t))$ as time goes on, where $T$ is the temperature. The Helmholtz free energy density is given by (Wang et al., 1993)

$$
\frac{f(T, \phi)}{L}=\frac{T}{T_{\mathrm{M}}}\left[\frac{3 \sqrt{2} \Gamma}{2 W} g(\phi)-\frac{T-T_{\mathrm{M}}}{T} p(\phi)\right] .
$$

Figure A.1 shows $f(T, \phi)$ as a function of $\phi$. The solid, dashed and dotted curves are for $T=T_{\mathrm{M}}$ (equilibrium), $T<T_{\mathrm{M}}$ (solidification), and $T>T_{\mathrm{M}}$ (melting), respectively. When $T=T_{\mathrm{M}}, f(T, \phi)$ has two minima at $\phi=0$ (solid) and $\phi=1$ (liquid). This stands for solid-liquid coexistence. When $T<T_{\mathrm{M}}$, the minimum at $\phi=0$ is lower than the (local) minimum at $\phi=1$. The difference in free energy between these two minima gives a driving force for solidification. On the contrary, when $T>T_{\mathrm{M}}$, the minimum at $\phi=1$ is lower than the (local) minimum at $\phi=0$, leading to melting of the solid phase.

The diffusion term of Eq. (1) makes the crystal-liquid interface smooth. This term represents the effect that the undulated interface in equilibrium with the parent liquid becomes flat. This effect is termed as the Gibbs-Thomson effect, namely, the equilibrium temperature at the curved interface is given by

$$
T_{\mathrm{N}}=T_{\mathrm{M}}(1-\Gamma / \rho),
$$

where $\rho$ is a local radius of curvature at a point of the interface (positive $\rho$ when convex toward the liquid). The term $T_{\mathrm{M}} \Gamma / \rho$ is the melting point depression due to the surface tension.

In PFM, the solid-liquid interface has finite thickness. The thickness $w$ is obtained from a steady-state solution of Eq. (1) at $T=T_{\mathrm{M}}$ (Wang et al., 1993). By dropping the time-derivative term and substituting $T=T_{\mathrm{M}}$, Eq. (1) can

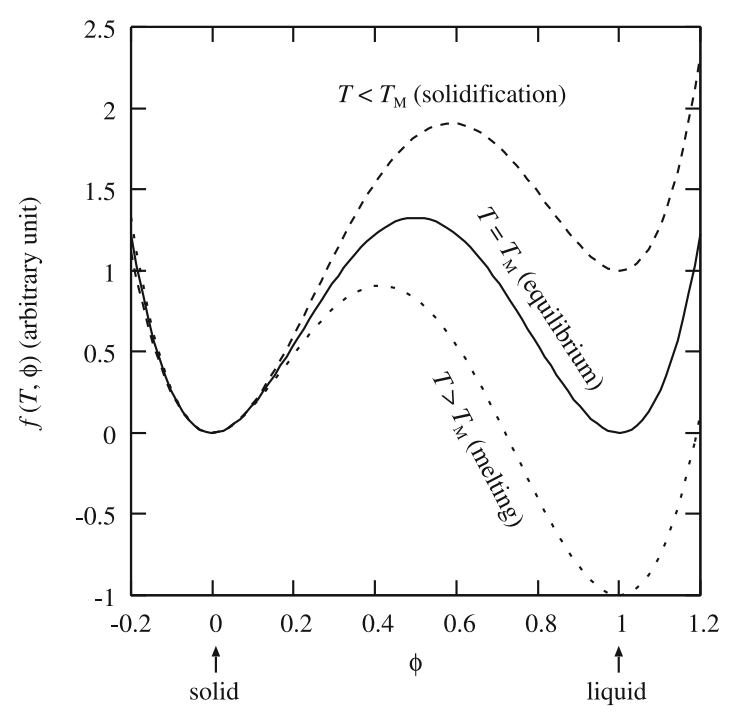

Fig. A.1. The helmholtz free energy density $f(T, \phi)$ adopted in PFM of Wang et al. (1993).

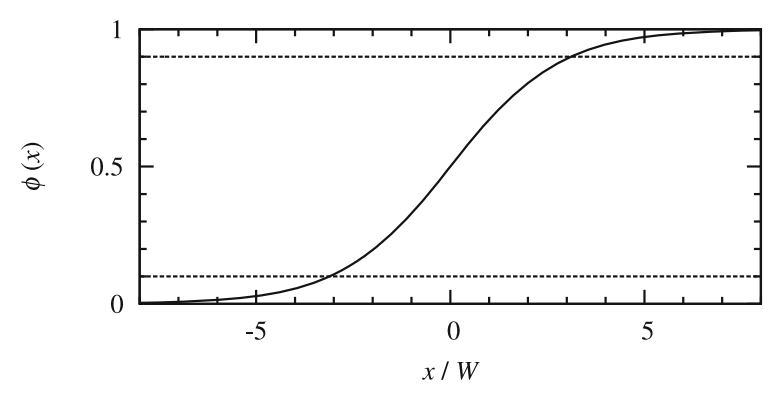

Fig. A.2. One-dimensional solution of Eq. (A.3) under equilibrium condition, $T=T_{\mathrm{M}}$.

be rewritten as

$$
\frac{\partial^{2} \phi}{\partial x^{2}}=\frac{1}{4 W^{2}} g^{\prime}(\phi) .
$$

The solution with boundary conditions $\phi \rightarrow 0$ as $x \rightarrow$ $-\infty$, and $\phi \rightarrow 1$ as $x \rightarrow+\infty$ is found to be

$$
\phi(x)=\frac{1}{2}\left[\tanh \left(\frac{x}{2 \sqrt{2} W}\right)+1\right],
$$

where we have chosen a constant of integration to locate the interface at $x=0$. Figure A. 2 shows this solution with respect to $x$. If we consider the solid-liquid interface being at $0.1 \leq \phi \leq 0.9$ (see Section 4.3), the width is found to be $w \approx 6 \mathrm{~W}$.

Wang et al. (1993) formulated the phase-field equation to solve the crystal growth velocity $V$ consistently with wellknown Wilson-Frenkel low in the limit of $W \rightarrow 0$. The Wilson-Frenkel low (Wilson, 1900; Frenkel, 1932) means that the growth velocity $V$ is proportional to the local supercooling, so we obtain

$$
V=\mu\left(T_{\mathrm{N}}-T\right)
$$

where the proportionality constant $\mu$ is called the kinetic coefficient and represents the efficiency for a growth unit to be incorporated into the crystal phase. Therefore, we can calculate the crystal growth velocity correctly by using a 
sufficiently fine computational mesh (Murray et al., 1995; Wang and Sekerka, 1996).

\section{References}

Amelin, T., A. N. Krot, I. D. Hutcheon, and A. A. Ulyanov, Lead isotopic ages of chondrules and calcium-aluminum-rich inclusions, Science, 297, 1678-1683, 2002.

Bi, Z. and R. F. Sekerka, Phase-field model of solidification of a binary alloy, Physica A, 261, 95-106, 1998.

$\mathrm{Bi}, \mathrm{Z}$. and R. F. Sekerka, Phase field modeling of shallow cells during directional solidification of a binary alloy, J. Crys. Growth, 237-239, 138-143, 2002.

Chalmers, B., Principles of Solidification, 319 pp, John Wiley \& Sons, Inc., USA, 1964.

Frenkel, J., Note on a relation between the speed of crystallization and viscosity, Phisik. Zeit. Sowjetunion, 1, 498-510, 1932.

Glicksman, M. E. and R. J. Schaefer, Investigation of solid/liquid interface temperatures via isenthalpic solidification, J. Crys. Growth, 1, 297-310, 1967.

Herlach, D. M., Non-equilibrium solidification of undercooled metallic metls, Materials Science and Engineering: R: Reports, 12(4-5), 177272,1994

Hewins, R. H., H. C. Connolly, Jr., G. E. Lofgren, and G. Libourel, Experimental Constraints on Chondrule formation, pages 286-316, Chondrites and the Protoplanetary Disk, San Francisco: Astronomical Society of the Pacific, 2005.

Jones, R. H., Petrology and mineralogy of Type II, FeO-rich chondrules in Semerkona (LL3.0): Origin by closed-system fractional crystallization, with evidence for supercooling, Geochim. Cosmochim. Acta, 54, 1785$1802,1990$.

Jones, R. H. and G. E. Lofgren, A comparison of feo-rich, porphyritic olivine chondrules in unequilibrated chondrites and experimental analogues, Meteoritics, 28, 213-221, 1993.

Jones, R. H., T. Lee, H. C. Connolly Jr., S. G. Love, and H. Shang, Formation of chondrules and CAIs: Theory vs. observation, in Protostars and Planets IV, pages 927-962, Univ. of Arizona Press, Tucson, 2000.

Langer, J. S. and H. Müller-Krumbhaar, Theory of dendritic growthI. Elements of a stability analysis, Acta Metallurgica, 26, 1681-1687, 1978.

Libourel, G., Systematics of calcium partitioning between olivine and silicate melt: implications for melt structure and calcium content of magmatic olivines, Contrib. Mineral. Petrol., 136, 63-80, 1999.

Lofgren, G. and A. B. Lanier, Dynamic crystallization study of barred olivine chondrules, Geochim. Cosmochim. Acta, 54, 3537-3551, 1990.

Lofgren, G. and W. J. Russell, Dynamic crystallization of chondrule melts of porphyritic and radial pyroxene composition, Geochim. Cosmochim. Acta, 50, 1715-1726, 1986.

Lux, G., K. Keil, and G. J. Taylor, Chondrules in h3 chondrites: textures, compositions and origins, Geochim. Cosmochim. Acta, 45(5), 675-685, 1981.

McFadden, G. B., A. A. Wheeler, R. J. Braun, S. R. Coriell, and R. F. Sekerka, Phase-field models for anisotropic interfaces, Phys. Rev. E, 48, 2016-2024, 1993.

Miura, H., E. Yokoyama, K. Nagashima, K. Tsukamoto, and A. Srivastava, Phase-field simulation for crystallization of a highly supercooled forsterite-chondrule melt droplet, J. Appl. Phys., 108, 114912, 2010.

Moriya, T., Glass Kougaku Handbook, 171 pp., Asakura Shoten, Tokyo, Japan, 1963 (in Japanese).

Murase, T. and A. R. McBirney, Properties of some common igneous rocks and their melts at high temperatures, Geol. Soc. Am. Bull., 84, 3563 3592, 1973

Murray, B. T., A. A. Wheeler, and M. E. Glicksman, Simulations of experimentally observed dendritic growth behavior using a phase-field model, J. Crys. Growth, 154, 386-400, 1995.

Nagashima, K., K. Tsukamoto, H. Satoh, H. Kobatake, and P. Dold, Reproduction of chondrules from levitated, hypercooled melts, J. Crys. Growth, 293, 193-197, 2006.

Nagashima, K., Y. Moriuchi, K. Tsukamoto, K. K. Tanaka, and H. Kobatake, Critical cooling rates for glass formation in levitated mg2sio4mgsio3 chondrule melts, J. Min. Petr. Sci., 103, 204-208, 2008.
Nelson, L. S., M. Blander, S. R. Skaggs, and K. Keil, Use of a co2 laser to prepare chondrule-like spherules from supercooled molten oxide and silicate droplets, Earth Planet. Sci. Lett., 14, 338-344, 1972.

Noguchi, T., Estimation of three-dimensional internal structures of some barred olivine chondrules in Allende (CV3) chondrite, Antarctic Meteorite Res., 15, 59-77, 2002.

Osada, Y. and A. Tsuchiyama, Experimental reproduction of rims in barred olivine chondrules by evaporation, Lunar Planet. Sci., 32, 1334-1335, 2001

Pack, A. and H. Palme, Partitioning of $\mathrm{Ca}$ and $\mathrm{Al}$ between forsterite and silicate melt in dynamic systems with implications for the origin of $\mathrm{Ca}$ Al-rich forsterites in primitive meteorites, Meteorit. Planet. Sci., 38, 1263-1281, 2003.

Pertermann, M. and A. M. Hofmeister, Thermal diffusivity of olivinegroup minerals at high temperature, Am. Mineral., 91, 1747-1760, 2006.

Radomsky, P. M. and R. H. Hewins, Formation conditions of pyroxeneolivine and magnesian olivine chondrules, Geochim. Cosmochim. Acta, 54, 3475-3490, 1990.

Sorby, H. C., On the structure and origin of meteorites, Nature, 15, 495498, 1877.

Srivastava, A., Y. Inatomi, K. Tsukamoto, T. Maki, and H. Miura, In situ visualization of crystallization inside high temperature silicate melts, $J$. Appl. Phys., 107, 114907, 2010.

Tanaka, K. K., T. Yamamoto, K. Nagashima, and K. Tsukamoto, A new method of evaluation of melt/crystal interfacial energy and activation energy of diffusion, J. Crys. Growth, 310, 1281-1286, 2008.

Tangeman, J. A., B. L. Phillips, A. Navrotsky, J. K. Richard, A. D. Hixson, and T. S. Key, Vitreous forsterite $\left(\mathrm{Mg}_{2} \mathrm{SiO}_{4}\right)$ : Synthesis, structure, and thermochemistry, Geophys. Res. Lett., 28, 2517-2520, 2001.

Tsuchiyama, A., H. Nagahara, and I. Kushiro, Experimental reproduction of textures of chondrules, Earth Planet. Sci. Lett., 48, 155-165, 1980.

Tsuchiyama, A., K. Uesugi, and T. Nakano, A study of three-dimensional structures of rocks and minerals using a high-resolution X-ray CT method-Primitive materials in the solar system and chondrules-. $J$. Geogr., 109, 845-858, 2000 (in Japanese).

Tsuchiyama, A., Y. Osada, T. Nakano, and K. Uesugi, Experimental reproduction of classic barred olivine chondrules: Open-system behavior of chondrule formation, Geochim. Cosmochim. Acta., 68, 653-672, 2004.

Tsukamoto, K., H. Satoh, Y. Takamura, and K. Kuribayashi, A new approach for the formation of olivine-chondrules by aeroacoustic levitation, Antarct. Meteorites, 24, 179-181, 1999.

Tsukamoto, K., H. Kobatake, K. Nagashima, H. Satoh, and H. Yurimoto, Crystallization of Cosmic Materials in Microgravity, Lunar Planet. Sci., 32, 1846-1847, 2001.

Uehara, T. and R. F. Sekerka, Phase field simulations of faceted growth for strong anisotropy of kinetic coefficient, J. Crys. Growth, 254, 251-261, 2003

Wang, S. L., R. F. Sekerka, A. A. Wheeler, B. T. Murray, S. R. Coriell, R. J. Braun, and G. B. McFadden, Thermodynamicallyconsistent phase-field models for solidification, Physica D, 69, 189-200, 1993.

Wang, S.-L. and R. F. Sekerka, Algorithms for phase field computation of the dendritic operating state at large supercoolings, J. Comput. Phys., 127, 110-117, 1996.

Watson, E. B., Surface enrichment and trace-element uptake during crystal growth, Geochim. Cosmochim. Acta, 60, 5013-5020, 1996.

Watson, E. B., A conceptual model for near-surface kinetic controls on the trace-element and stable isotope composition of abiogenic calcite crystals, Geochim. Cosmochim. Acta, 68, 1473-1488, 2004.

Weisberg, M. K., Barred olivine chondrules in ordinary chondrites, $J$ Geophys. Res., 92, E663-E678, 1987.

Wilson, H. A., On the velocity of solidification and viscosity of supercooled liquids, Philos. Mag., 50, 238-250, 1900.

$\mathrm{Xu}$, J. J., Interfacial Wave Theory of Pattern Formation, 174 pp., SpringerVerlag, Berlin, Germany, 1998.

H. Miura (e-mail: miurah@m.tohoku.ac.jp), E. Yokoyama, K Nagashima, K. Tsukamoto, and A. Srivastava 